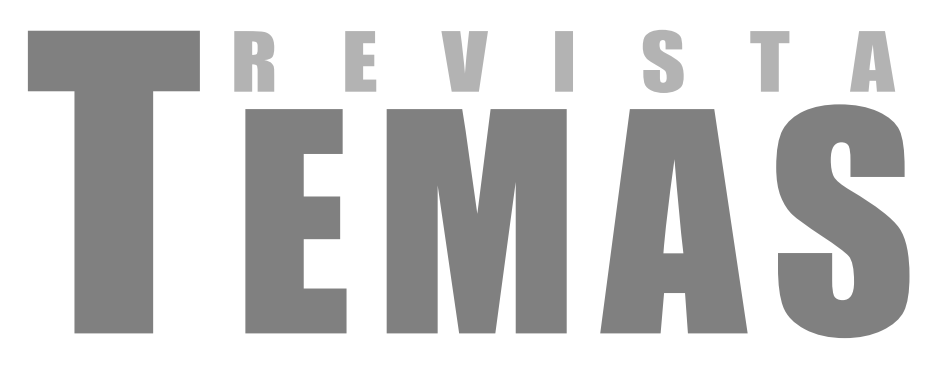

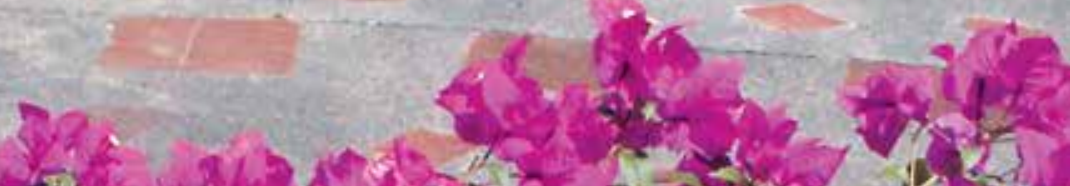

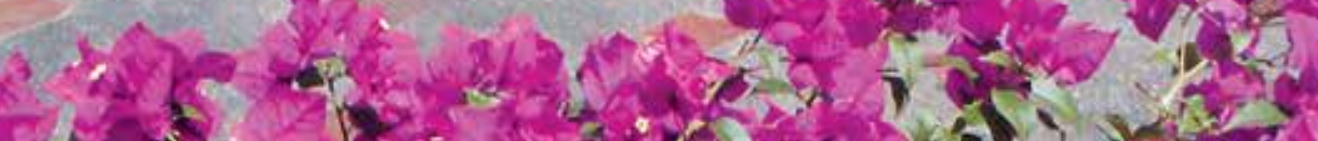

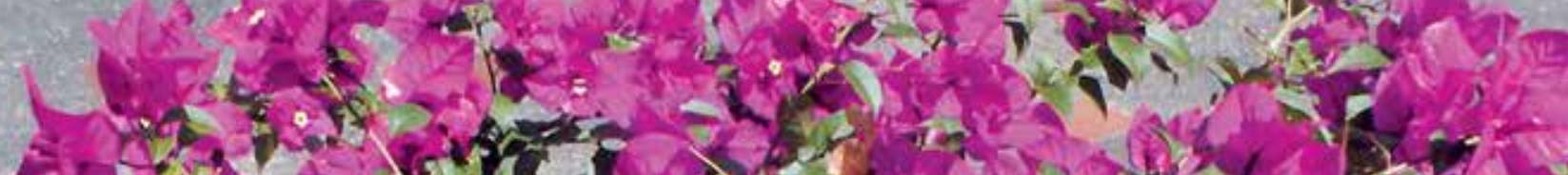

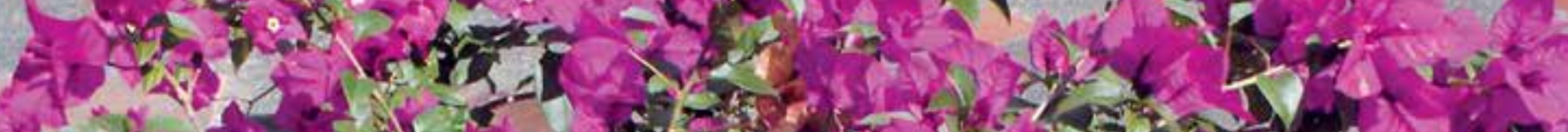
8.

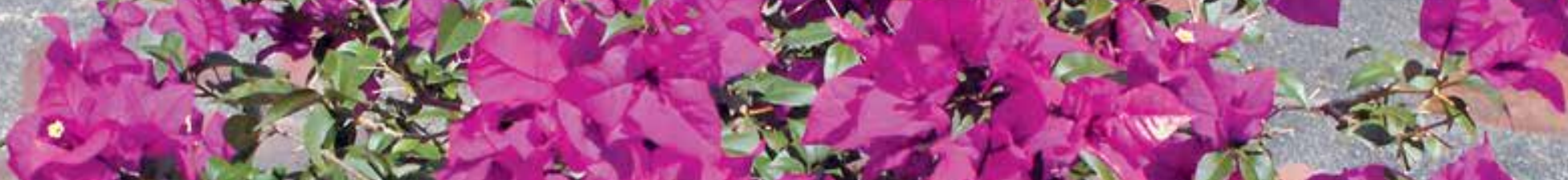

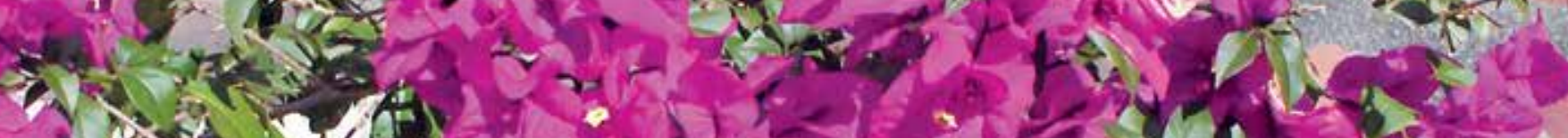

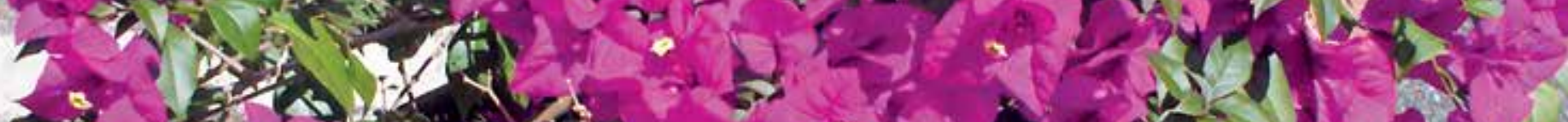

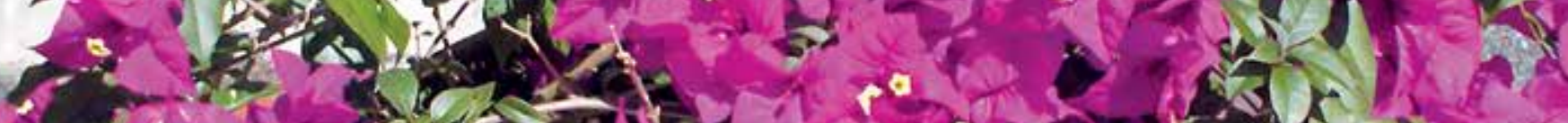

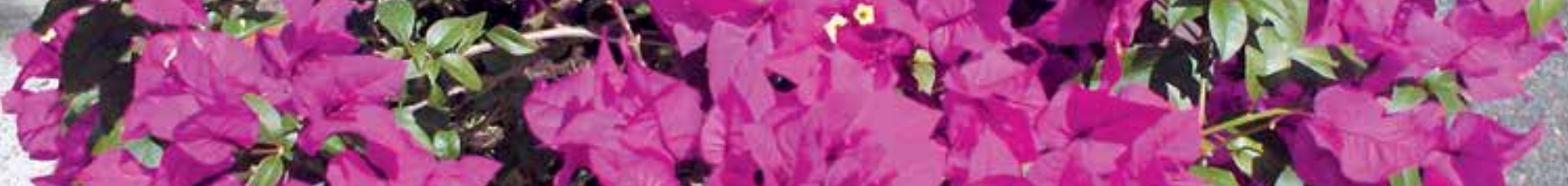

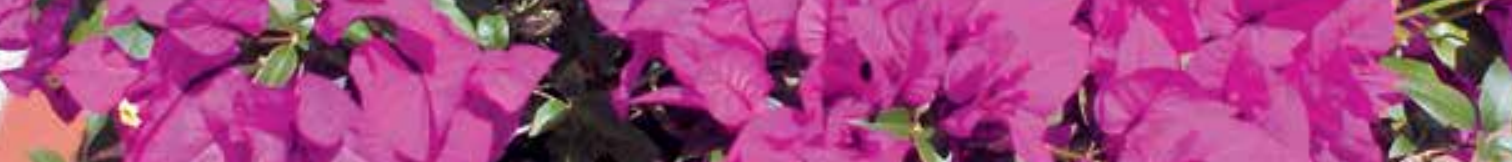

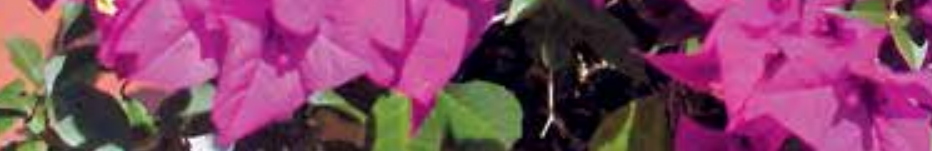

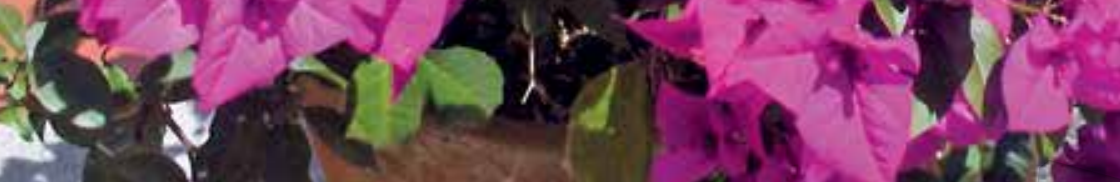
$-2)^{2}$ का 

Referencia al citar este artículo:

Peña, L.D. (2016). Hábitos de consumo de las mujeres bumanguesas y ecofeminismo en relación con el posconflicto. REVISTA TEMAS, 3(10), 183-194

\title{
Hábitos de consumo de las mujeres bumanguesas y ecofeminismo en relación con el posconflicto
}

\author{
Leidy Dayanna Peña Hernández ${ }^{2}$
}

Recibido: $4 / 30 / 2016$

Aceptado: 8/7/2016

\section{Resumen}

Se analizan los hábitos de consumo de las mujeres bumanguesas, a través de un estudio etnográfico, desde el ámbito de la edad, ingresos y los bienes que compran con frecuencia, realizando inicialmente un contexto económico sobre la economía lineal, para posteriormente poder abarcar el ecofeminismo, el cual será clave para las alternativas de solución a la economía lineal y dentro de un entorno de posconflicto, donde las mujeres pueden aportar para la construcción de la paz. Así mismo, se evalúan los diferentes actores en un análisis intersectorial, para determinar la importancia y la interdependencia de cada uno de ellos dentro del mismo sistema.

\section{Palabras clave}

Consumo, mujeres, ecofeminismo, posconflicto.

\section{Consumption habits of bumanguesas women and ecofeminism regarding the post-conflict}

\begin{abstract}
It is an analysis of the consumption habits of bumanguesas women, through an ethnographic study from an socioeconomic scope and their frequently shopping. First, I did an economic context about lineal economic, then, I wrote about ecofeminism because, this is the key for alternatives solutions in the lineal economic and within a post-conflict environment, in where, women can bring different options to peace building. Likewise, I evaluated the roles of different actors in a cross-sectorial analysis, with the intention to determinate your importance and independence of each one, in a same system.
\end{abstract}

\section{Keywords}

Consumption, women, ecofeminism, posconflict.

\footnotetext{
1 Artículo de reflexión.

2 Estudiante-investigadora-tutora PAAI del Programa de Negocios Internacionales de la Universidad Santo Tomás. Correo electrónico: leidyd1220@ hotmail.com
} 


\section{Introducción}

El consumo ha tenido un crecimiento exponencial durante los últimos años en Colombia, a raíz de la apertura económica en 1991, durante el gobierno de César Gaviria. Es por dicho crecimiento que se hace necesario estudiar con mayor determinación este fenómeno, pues claramente tiene una relación directa con el medio ambiente y el cuidado que le damos. Sin embargo, después de 1934, se le otorga a las mujeres un título de "compradoras compulsivas", ya que fue en ese año que la mujer logró hacer compras a su nombre propio, pues con anterioridad a esto, aunque ella hiciera las compras, los bienes quedaban a nombre de su padre en caso se ser soltera, o a nombre de su esposo.

Con la situación anterior, se logra visualizar la lucha que las mujeres han tenido a lo largo de la historia para que le sean reconocidos y respetados sus derechos como seres humanos y como mujeres, para que actualmente el género femenino logre participar en igualdad de condiciones que los hombres en los diferentes ámbitos de la vida, y aun hoy, se sigue la lucha a través de un movimiento social llamado feminismo, pues aunque se han alcanzado grandes avances, el cambio cultural y de pensamiento es una transición que lleva mucho tiempo.

Seguramente cuando la gente piensa en la paz, erróneamente se cree que es una situación inmediata, sin considerar todos los factores y actores que hacen parte de esta complejidad y, por dicho motivo, he considerado abarcar el posconflicto desde un ámbito civil y cotidiano.

Es por esto, que sobran razones para involucrar el posconflicto colombiano con el consumo de las mujeres y el ecofeminismo, ya que es un escenario para el cual toda Colombia debe estar preparada, para afrontar este nuevo cambio en su sociedad, pues es algo inminente con las actuales negociaciones de paz entre el actual presidente Juan Manuel Santos y las FARC, que está siendo vista y apoyada desde el marco internacional.

Bajo este escenario, el objetivo de este artículo es analizar los hábitos de consumo de las mujeres bumanguesas, con el fin de determinar la importancia de su consumo en términos medioambientales y de posconflicto.

\section{Metodología}

Para lograr el objetivo anteriormente propuesto, se lleva a cabo una metodología de tipo documental con un carácter analítico-descriptivo, con la cual se estudian teorías y aportes en las diferentes temáticas que se abarcan en el presente artículo.

La búsqueda de la información de documentos, artículos y libros se realiza a través de las siguientes bases de datos: Business Source Complete -EBSCO-, Academic Search Complete, E-libro, LSE Research Online, entre otros.

En cuanto el análisis de los hábitos de consumo de las mujeres bumanguesas se hizo pertinente realizar un estudio etnográfico $^{3}$ en el cual se visualicen las principales compras que realizan las mujeres, el gasto o presupuesto destinado para su consumo y la frecuencia de sus compras. Cabe señalar que dicha investigación se centra principalmente en bienes que se compran con el dinero que no está destinado a alimentación, arriendo, servicios públicos, entre otros. Esto se hará más específico cuando se estén realizando los análisis pertinentes.

Dicho estudio etnográfico es una investigación de campo cualitativa, la cual

3 Estudio etnográfico realizado bajo mi autoría en el mes de septiembre del año 2014, con 500 cuestionarios resueltos. 
se realizó en Bucaramanga, a través de herramientas, como:

- La observación participante: para registrar los comportamientos de consumo de las mujeres de acuerdo con su edad, y preferencias con base en las publicidades.

- Conversaciones: mientras hablaba y compartía con otras mujeres, les preguntaba aspectos relacionados con sus compras, para obtener información sobre porcentajes de sus ingresos que reservan para el gasto en su consumo fuera de las necesidades básicas.

- Cuestionario: estructurado con solo tres preguntas, el cual le realizaba a las mujeres que se veían dispuestas en las conversaciones y que concedían su permiso para el uso de sus respuestas en este estudio, ya que con el cuestionario se logra obtener datos cuantitativos, muy importantes para este artículo.

Con el fin de lograr la metodología propuesta, se han especificado las siguientes fases:

1. Estudio etnográfico y análisis de los resultados obtenidos en este.

2. Identificación de la información pertinente al consumo, ecofeminismo y posconflicto.

3. Confrontación, análisis e interpretación de la información recolectada.

4. Elaboración de las conclusiones y recomendaciones finales.

\section{Economías lineales}

Para abordar la temática del consumo conviene explicar la economía lineal, desde su aspecto económico, para lo cual es necesario comprender que es un proceso con un orden lógico, dado de la siguiente manera:
Extracción de los recursos naturales, transformación y producción con dichos recursos, distribución de los productos, consumo de los bienes y finalmente el descarte/desecho de estos (Belz, Peattie, \& Galì, 2013).

Este sistema lineal ha sido empleado de generación a generación, en un planeta con recursos limitados, destacando que se usan tanto recursos renovables como no renovables, y de acuerdo con un informe entregado por Estados Unidos y desarrollado por científicos, se afirma que se ha reducido la vegetación del planeta en 2,3 millones de kilómetros cuadrados de forestas entre el año 2000 y 2012.

Esto se relaciona con la economía lineal, y por ello se argumenta que el sistema está mal aplicado, no solo por desempeñarse en un planeta finito, sino también porque la pérdida de forestas "modifican muchos aspectos importantes del ecosistema, incluida la regulación del clima, el almacenamiento de carbono, la biodiversidad y los suministros de agua" (Rpp noticias, 2013).

Estos cambios, no solo son de interés para los ecologistas, también son de interés para la población que se ve afectada, e incluso son interés para las empresas, como es en el caso del clima para sus producciones, o de la huella de carbono que está relacionada con la responsabilidad social que las empresas tienen. Es por esto último, que en este artículo, necesariamente se relacionarán a las empresas, pues estas realizan grandes esfuerzos en incrementar el consumo por parte de sus clientes, olvidando en ocasiones la responsabilidad que tiene con la sociedad y su entorno.

\section{Ecofeminismo}

El ecofeminismo nace como término en los años setenta del siglo $X X$, debido a la súper población que se presentaba en el momento y por lo cual se otorgan 
los derechos sexuales y reproductivos; razón por la cual el concepto surgió del encuentro entre la ecología y el feminismo (Puelo, 2008).

Esto ha propiciado nuevos fenómenos como el de control natal que ha generado un importante lugar en la economía en torno a las prácticas del ecofeminismo. Las farmacéuticas con la producción y venta de anticonceptivos y métodos de planificación sexual, han ganado gran participación en el mercado. Además se les otorga la decisión de compra de estos artículos a las mujeres, es decir, se les da una mayor autonomía sobre su cuerpo.

Algo más hay que añadir, y es una aclaración con respecto al concepto ecofeminismo, pues "mujeres y ecología no son sinónimos" y aun así "El colectivo femenino ha desarrollado una subjetividad relacional, atenta a los demás con mayor expresión de la afectividad" (Puelo, 2008).

No obstante, es válido aportar desde la teoría que cuando el concepto ecofeminismo recoge la palabra feminismo, esta no hace referencia solo al género o sexo de la persona, pues hay que tener en cuenta que las actitudes y aspectos femeninos se dan tanto en hombres como en mujeres, es decir, puede existir un hombre con pensamiento feminista sin necesariamente tener una orientación sexual diferente a la heterogénea. Es por esto que, enfatizo que en aspectos ecológicos la responsabilidad es de todos, y esto no incluye solo personas naturales sino también entes jurídicos, incluso sociedades enteras.

Una vez, hecho este aporte, se proseguirá con la posición de las mujeres, pues es esto lo que ha estado marcado y definido a través del tiempo.

Este concepto es necesario para nuestro análisis, puesto que la teoría del ecofeminismo plantea un reencantamiento del mundo natural para devolverle la dignidad que le ha sido arrebatada, así como denunciar las nuevas formas de colonización que afectan a los países del Sur ; lo cual está siendo promovido por las mujeres, ya que esto les brindará un mayor empoderamiento y reconocimiento frente a los estereotipos y prejuicios impuestos por la sociedad en la cual se desenvuelven por los roles que fueron distribuidos por sus antepasados (Puelo, 2008).

Como se planteó al inicio del artículo, se deben analizar los hábitos de consumo de las mujeres bumanguesas, objeto de este estudio, y mirar la relación que estos hábitos tienen con referencia a la ecología, desde el enfoque de explotación de recursos naturales por parte de las empresas con justificación en producir los bienes que "necesitan las mujeres" que posteriormente compraran, usaran y desecharan, sin ningún tipo de garantía ni cuidado hacia el entorno en el que habita la raza humana.

Aquí he de referirme también, que las mujeres desde la base social pueden desarrollar grandes cambios y avances para mitigar el problema medioambiental a través del cambio fundamentado en una consciencia crítica y espiritual sobre sus hábitos de consumo.

Un movimiento que argumenta esta idea, es el movimiento de mujeres Chipko, desarrollado en la India, en contra del "mal desarrollo" del cual hablamos en la economía lineal, con base en un sistema capitalista. Con estos movimientos bien organizados se pueden destacar impac-

4 Colonización, desde la perspectiva del poder que ejercen Estados extranjeros en un territorio fuera de sus fronteras para extraer sus recursos naturales, explotar el trabajo de su población y dominar a través de las relaciones asuntos de carácter administrativo, militar y económico dentro de dicho Estado. Enfocados en un ámbito actual de la globalización, donde los países del primer mundo, dominan a los del tercer mundo. Para colocarlo en un contexto real, Estados Unidos está "colonizando" a Colombia, a través del dominio y la interdependencia que tiene Colombia de este país, por lo cual EE. UU. presiona y condiciona ciertos aspectos administrativos, militares y económicos de Colombia. 
tos fuertes sobre este sistema, como lo redacta María Medina Vincent (2013), en la evolución del ecofeminismo:

A raíz de este primer movimiento, las reclamaciones se han extendido a diversos ámbitos, por ejemplo, las mujeres adivasi de la India (Shiva, 2005) consiguieron en el 2004 cerrar una fábrica de la empresa Coca-Cola en Plachimada, porque ésta contaminaba los acuíferos de la zona y estaban acabando con los medios de vida de la población autóctona, obligando a las mujeres a desplazarse varios kilómetros en busca de agua.

Si todo esto se puede realizar con una comunidad de mujeres, que tiene dificultades para organizarse y demás, ¿Cuánto más se puede realizar desde las decisiones de compras que tiene cada mujer? Si cada mujer que reside en Bucaramanga pasa sus posibles compras por un análisis racional sobre la necesidad del mismo, habría muchos productos que no se comprarían porque realmente no tenemos la necesidad de adquirirlos y solo con eso, los logros, avances y preservación de la naturaleza aumentarían de gran manera. Por ello, a continuación, realizaremos un análisis mucho más detallado de los resultados del estudio etnográfico realizado previamente.

\section{Hábitos de consumo de las mujeres bumanguesas, de acuerdo con un estudio etnográfico}

La primera parte del estudio etnográfico fue realizado a través de la herramienta de la observación, donde estuvieron involucradas mujeres entre 15 y 60 años de edad, las cuales tienen grandes diferencias marcadas en sus hábitos de consumo con respecto a la diferencia de edad, ya que en el caso de las mujeres mayores se logró determinar que no se dejan influenciar mucho por las publicidades y comerciales, en realidad lo que buscan es calidad en los productos que adquieren, adicional compran cosas que les guste puesto que ellas tienen totalmente la decisión de compra. Contrariamente a las mujeres jóvenes que son mucho más sensibles a las publicidades y desean comprar lo que está en tendencia, desean adquirir lo nuevo, estar a la moda.

Por tal motivo, las mujeres jóvenes tienen un mayor consumo, debido a que están cayendo en la trampa de la obsolescencia perdida ${ }^{5}$ y en la inevitable programada ${ }^{6}$, las cuales al tenerlas en cuenta podemos darnos una idea del por qué estamos usando demasiadas cosas, iel sistema nos lo manda, el sistema lo exige!

De estas circunstancias nace el hecho de que se afecte directamente a cada país, no solo por contribuir con el sistema económico que ha sido planteado y diseñado para que funcione de esta manera, sino también lamentablemente por la acumulación de las basuras; basuras de las que no todos los países tienen un buen tratamiento. Desde este punto de vista se debe visualizar el planteamiento ambiental que está inherente a dicha problemática del consumo.

Llegado a este punto, pasare a la segunda parte del estudio etnográfico realizado a través de la herramienta de las conversaciones, con las cuales se lograron determinar los siguientes resultados, sobre el porcentaje de sus ingresos que dedican al consumo de los bienes que no hacen parte de las necesidades básicas.

Por simplicidad, podemos expresar que el porcentaje dedicado a otros bienes de consumo varían y son directamente relacionados con el nivel socio-económico de las mujeres en cuestión, veamos cómo se desenvuelve:

5 Obsolescencia perdida, la cual por medio de la publicidad nos dice lo que debemos hacer, por qué está bien y las nuevas tendencias que marcan la moda. Estas incitan a comprar de manera desmedida.

6 Obsolescencia programada, referenciando al producto diseñado para ser desechado, es decir, los productos son producidos con una vida útil ya definida en tiempo de uso. 
En promedio, las mujeres que son solteras y viven con sus padres o en una pieza en arrendamiento, y que ganan un salario mínimo legal, dedican solo el $18 \%$ de sus ingresos en otros bienes que realmente no necesitan, mientras que las mujeres que están en una relación sentimental (unión libre o casadas) y ganan este mismo valor, dedican menos del $10 \%$ a otros bienes de consumo.

Gran diferencia encontrada, con las mujeres que viven en situación similar pero que ganan más del salario mínimo (aproximadamente entre 2 y 5 salarios mínimos), pues estas dedican entre un $38 \%$ y $43 \%$ de sus ingresos en otros bienes de consumo.

Mientras que las mujeres que se encuentran pensionadas alcanzan un disponible de hasta un $\mathbf{7 2} \%$ para realizar compras de bienes que no necesitan, pero que usan para su ocio, sin embargo, es de aclarar que suelen gastar gran parte del $72 \%$ en servicios de turismo y en bienes con marcas posicionadas en ellos y que no han tenido grandes cambios, pues hacen parte de la generación Baby boomers ${ }^{7}$ las cuales tienen un pensamiento de disfrutar lo que han ganado o han conseguido por trabajar toda su vida sin tener en cuenta mucho la tecnología (Osorio, 2013). Este porcentaje es muy impactante, pues solo utilizan el $28 \%$ para bienes necesarios, esto es debido a que sus obligaciones a una edad avanzada disminuyen $y$, generalmente, ya tienen su casa propia, o viven con alguno de sus hijos, quien aporta la mayoría de gastos esenciales que la mujer necesita. Este escenario también influye sobre las compras que realizan las mujeres comprendidas en esta categoría, pues el $29 \%$ de los bienes que compra innecesariamente, lo

\footnotetext{
7 La generación de los "Baby boomers" es reconocida mundialmente como la mayor generación en volumen de todos los tiempos, con la explosión de natalidad. Las personas denominadas de esta generación son las nacidas entre 1946 y 1964, tras la Segunda Guerra Mundial.
}

hace para dar regalos a sus familiares, en especial a los nietos que tienen cerca.

Una vez definida la cantidad de ingreso que las mujeres presupuestan o gastan en bienes de consumo no necesarios, es imprescindible determinar cuáles son los bienes que ellas compran frecuentemente. Esto nos ayudará a determinar una posición de las empresas productoras y distribuidoras de dichos productos, y con ello podremos dar las recomendaciones pertinentes.

Figura 1. Descripción de los productos que compran las mujeres bumanguesas entre los 15 y 60 años de edad, con ingresos de un salario mínimo hasta cinco salarios mínimos y mujeres pensionadas

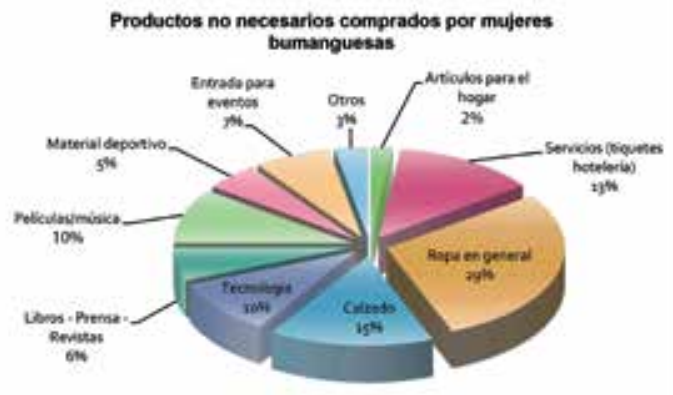

Aquí se destaca que el mayor porcentaje de las compras realizadas por mujeres está enfocados en la ropa, con un $29 \%$, seguido por el calzado, un $15 \%$; pero si observamos con cuidado nos damos cuenta que los libros, prensa, y revistas solo tienen una participación del $6 \%$, será que ese $6 \%$ corresponde a los ingresos que dedicamos al estudio, o el estudio está comprendido en la categoría otros, con un $3 \%$, esta pregunta reflexiva es formulada teniendo en cuenta que el estudio abarca mujeres en edad para formarse técnica o profesionalmente; una posible hipótesis relacionada con esto es que las mujeres que estudian lo hacen porque sus padres pagan su educación, y que las mujeres independientes que estudian, consideran la educación un servicio necesario que debe adquirir y por ello lo excluye de este cuestionario. 
Este es un interesante análisis en el cual no me voy a centrar, porque solo eso nos llevaría a otra investigación especializada sobre el tema.

Continuare, con los servicios turísti$\cos (13 \%)$ y los productos tecnológicos $(10 \%)$, los cuales tienen un carácter fundamental para las empresas que ofrecen este portafolio a sus clientes. Pues los servicios turísticos son de fundamental importancia, pues estos generan un impacto en los ecosistemas y es responsabilidad de las empresas garantizar que se evite al máximo dañar el entorno del lugar donde se prestan los servicios. De acuerdo con Figueredo y Rozo de la Universidad Externado de Colombia:

La regulación que debe enfrentar el Estado debe considerar no solo la protección de los ecosistemas, sino advertir de los posibles impactos y destinar los recursos para atender costos por contaminación y en lo posible trasladarlos a los responsables: 'el que contamina paga' (2002).

Por otra parte, los productos tecnológicos ofrecidos en la actualidad son los bienes que tienen mejor definida la obsolescencia programada, haciendo la vida útil de los productos cada vez más corta, además de que los bienes tecnológicos avanzan rápidamente -aunque sea solo con un mínimo cambio- lo cual influye en las personas para que se estén actualizando.

Y lo que realmente acontece aquí, es el proceso de producción que se lleva a acabo para este tipo de bienes, pues es uno de los sectores que proporciona mayor contaminación, a causa de sus procesos industriales.

Ha llegado el momento de analizar a fondo estos hábitos de compras, pues no solo se trata de determinar qué compran frecuentemente, sino identificar por qué lo hacen, cuál es la causa y la motivación de las mujeres para adquirir los bienes expuestos anteriormente. Para esto realizaré un análisis intersectorial, estudiaré los comportamientos del consumidor y evaluaré la psicología que las empresas usan en sus publicidades.

\section{Análisis intersectorial}

El análisis intersectorial tiene como objetivo revelar las variadas identidades, exponer los diferentes tipos de discriminación y desventaja que se dan como consecuencia de la combinación de identidades (Assosiation for Women's Rights im Development, 2004).

Hemos examinado hasta aquí los múltiples actores que están relacionados de alguna manera con el consumo de las mujeres, tales como las empresas, el Estado, el medio ambiente y por supuesto la sociedad del género femenino. Mas no se trata tan solo de mirarlos a cada uno en su posición, pues todo está conectado y hace parte del sistema económico.

Aquí conviene detenerse un poco a fin de explicar esta conexión. Veamos, el medio ambiente provee de recursos naturales a las empresas, las cuales los transforman y venden a los clientes, dichos clientes en este caso son las mujeres, quienes los usan y posteriormente desechan, por lo cual los productos ahora hacen parte de las basuras de la sociedad, y aquellas basuras están o vuelven de alguna manera al medio ambiente y lo afecta de manera negativa, sin embargo, para brindar un mejor tratamiento a las basuras y evitar que impacte tan negativamente al ambiente, está el Estado, que financia estas actividades del tratamiento de las basuras a través de los impuestos que le son cobrados a las personas que compraron los productos, así como a las empresas que los fabricaron; pero el Estado también se encarga de tener unas regulaciones para la buena convivencia de todos los actores, entre lo cual, pueden 
estar las reglamentaciones para evitar que tanto empresas como personas maltraten el medio ambiente, o en su defecto cobrar por los daños causados y su "reparación".

Con esta relación en mente, se visualiza que cada actor tiene una identidad y función específica. Lo curioso es que al cambiar el ángulo de la perspectiva y enfocarnos en las mujeres, vemos que solo ellas ya tienen múltiples identidades, y que ninguna es igual en su totalidad a otra, pero para la tranquilidad del lector y de los investigadores, las mujeres (o las personas en general) se pueden "clasificar" o segmentar para el caso del marketing. Estas clasificaciones se pueden llevar a cabo por diversas variables, pero en todo caso, facilitan los estudios que se realizan.

Para ilustrar mejor este aspecto, plantearé la pregunta que me ha traído a realizar el análisis intersectorial: ¿Las mujeres consumimos para no ser discriminadas?, ¿lo hacemos porque nos afecta ser discriminadas por características psicológicas propias de la feminidad, como la sensibilidad? Bueno, en un primer plano, conviene aclarar que la sensibilidad es un estereotipo que se les asigna a las mujeres, porque se cree que el hombre no tiene este sentido sensible, pero la idea aquí es, que no todas las mujeres son sensibles y no todos los hombres carecen de sensibilidad.

Pero el caso es la discriminación, porque como hemos hablado anteriormente, los hábitos de consumo de las mujeres dependen de su poder adquisitivo, es decir, de sus ingresos, y el qué compran está relacionado a sus experiencias individuales y únicas. Mirándolo así, quizás, entonces no habría posibilidad de marginalizar a otras personas por lo que compran.
Lo que Bourdieu plantea en el concepto de habitus ${ }^{8}$ es que "al elegir en el espacio de los bienes y los servicios disponibles proyectamos la posición que ocupamos en el espacio social" (Guerra, 2010), esto nos lleva a pensar que los comportamientos de consumo son dados por una psicología en el estatus económico de la mujer; para explicarme mejor, una mujer de un estrato alto no quiere ser confundida con una mujer de estrato inferior, y por ello compra artículos que tengan un carácter exclusivo o de reputación superior frente a otros artículos.

Esto sucede mucho en productos tecnológicos, ya que entre más actual sea la tecnología que la persona usa, mayor estatus le brinda frente a los demás; o por el contrario, si la mujer es la única en su círculo de amigas que tiene una gama baja en la tecnología, las demás presionaran para que esta mujer suba al nivel económico que tienen sus amigas. $O$ en el caso del sector textil y de confecciones, donde las mujeres de la "alta sociedad" usan ropa de diseñador hechos a la medida, mientras las demás mujeres compran en almacenes de cadena y locales comerciales.

Todo esto parece confirmar la brecha que hay entre las mujeres y sus hábitos de consumo de acuerdo con su nivel económico. La noticia es que en la ciudad de Bucaramanga, estos aspectos son más llevaderos, flexibles y suaves, dado que la discriminación no está tan marcada con respecto a criterios de compra.

\section{Comportamientos del consumidor}

El comportamiento del consumidor está ligado con la situación del entorno, por ejemplo, con la apertura económica de Colombia, se realizaron grandes cambios que lograron hacer de la sociedad

8 Habitus, precisa Bourdieu a partir de la distinción, es a la vez un sistema de esquemas de percepción y de apreciación de las prácticas (Guerra, 2010, p. 391). 
un consumo de masas, para los cuales las publicidades son dirigidas con características funcionales de los productos (Santamarina y Marinas, 1994).

Avanzando en el tiempo encontramos que el comportamiento del consumidor ha evolucionado y los productos también han tenido que hacerlo para seguir el ritmo de sus demandantes, pues en la actualidad son mucho más exigentes en cuanto a lo que quieren y como lo quieren.

Sin embargo, las empresas han ayudado mucho en las exigencias del consumidor, pues les han creado unas necesidades que realmente no tienen; es por esto que "el sentimiento de que se necesita más de lo que se gana es más fuerte que nunca" (Borrás, 2000). Análogamente, el consumidor tal y como los señala Keynes: "consume en función de su renta y establece su preferencia en función de los precios".

Por otra parte, el comportamiento está influenciado por las emociones del individuo y de allí el argumento que las mujeres son quienes más compran, guiadas por el impulso y las emociones. Esta condición, la han fortalecido las empresas a través de sus publicidades.

El libro de psicología económica y del compartimiento del consumidor basa el comportamiento del consumidor en lo que representa el producto y no lo que es en sí, haciéndolo de la siguiente manera:

Realmente el mercado de hoy es un mercado dominado por lo simbólico. El valor signo viene a superar y desplazar al valor de uso y al valor de cambio, ya que estos están dominados por la lógica de significación social.

Adviértase, pues que el consumidor también tiene unas fases en su comportamiento al realizar una compra, las cuales el merchandising las separa en cuatro etapas: (a) el cliente mira (b) el cliente siente (c) el cliente examina (d) el cliente compra (Prieto, 2011). Vale la pena aclarar que estas cuatro se dan cuando el cliente tiene un contacto directo con el producto. Adicional, dichas compras pueden llevarse en diferentes contextos y por ello hay diferentes tipos de compras, tales como las previstas (comportamiento racional), las impulsivas (comportamiento irracional), y la compra impulsiva pura, la cual representa el $14 \%$ de las compras totales (Prieto, 2011)

\section{Psicología en las publicidades}

La publicidad que lanza una empresa es fundamental para dar a conocer su producto y realizar un posicionamiento de la marca, por lo cual, la empresa debe conocer a profundidad su negocio y el segmento al cual está dirigido. Esto, porque una vez tiene identificado su nicho de mercado, ya tiene una clasificación y con ello un perfil psicosocial del consumidor.

Es precisamente el análisis anterior, el que le permitirá a la empresa planear y ejecutar sus estrategias usadas en las publicidades, pues recordando la obsolescencia perdida de la cual hablamos al inicio de este artículo, es que entendemos que hay todo un estudio detrás de una propaganda televisiva, de radio, en volantes, entre otras. Por medio de las cuales se nos da órdenes que nuestro subconsciente es capaz de comprender, aun y cuando nosotros pensamos que es un simple aviso de publicidad.

El gran secreto de este aspecto son las emociones con las que cotidianamente vive el ser humano (García y Thomas, 2011) y, en este caso, con las emociones y situaciones que experimentan las mujeres, de tal manera, que logran hacer que la mujer se identifique con la publicidad y sienta gran empatía con el producto, hasta el punto que desee comprarlo, aunque no lo necesite. 
Por supuesto, esto está muy ligado a la cultura, vamos al caso colombiano: la gente en ocasiones prefiere comprar un producto importado, porque se tiene la creencia que lo de afuera es mejor que lo nuestro, por diferentes causas, la más relevante, a veces olvidamos apreciar lo nuestro, y valorar lo que hacen las manos colombianas.

Es que esta noción está basada en el comportamiento del consumidor, en la que prefiere comprar un producto por su valor simbólico, tal y como se expresa en el siguiente apartado.

Y para ello en la publicidad se utilizan marcas con denotaciones extranjeras, ya que la primacía de estas marcas proviene más bien, de la asociación entre marca y modo de vida, ambos de origen extranjero (Borrás, 2000).

¿Recuerdan cuando les hablaba sobre la colonización que afecta a los países del Sur? Bueno, este es otro ejemplo sobre ello, o será que ¿Estados Unidos no nos está colonizando con sus marcas?

\section{Posconflicto}

Paralelamente a todo lo que ya he expuesto en las páginas anteriores, hay que señalar que en Colombia se ha vivido un conflicto armado por más de 50 años, que está a punto de terminar con la firma de la paz que están negociando el Gobierno de Juan Manual Santos con las FARC en la Habana, Cuba.

Cabe señalar que lo más importante de este acuerdo de paz no está en la firma del mismo, sino en ¿qué hacer y cómo afrontar los cambios que vienen?, pues el proceso requiere de tiempo para asumir todas las transiciones que la sociedad debe proseguir.

En primera medida, es necesario decir que al finalizar el conflicto, todas aquellas personas que estaban directamente involucradas en este deberán retirarse, en el caso de quienes hayan cometido crímenes de lesa humanidad, indiscutiblemente deberán pagar con cárcel, y los demás podrán reintegrarse a la vida civil, así que la sociedad deberá estar preparada para recibirlos, teniendo en cuenta que en estas personas reintegradas habrá mujeres. En este sentido, las empresas pueden brindar oportunidades de capacitación para dichas personas, con la intención de que se califiquen para realizar alguna actividad dentro de las mismas empresas.

Para pensar en lo que debemos hacer, se deben entender los inicios de cualquier conflicto, para ello recordemos que se pueden remontar a las desigualdades, carencias, ambición, competencia, poder, entre otros, sobre todo en justificaciones relacionadas con las tierras, recursos naturales y recursos económicos.

Recordando que estos recursos naturales están implicados en las economías lineales, por lo cual se hace necesario que se realicen cambios en el sistema en que se manejan los recursos, cambiando hacia una nueva alternativa mucho más amigable con el medio ambiente, como son las economías circulares o cíclicas con el fin de lograr un desarrollo sostenible, el cual se plantea como una ventaja competitiva.

Adicional a esto, las mujeres pueden ayudar a la construcción de paz a través de sus decisiones de compras, adquiriendo los bienes de aquellas empresas que les brindan oportunidades a quienes se reintegran, y a su vez, evitando consumir y desechar rápidamente los bienes no necesarios que se ubiquen dentro de la economía lineal.

Por otra parte, se debe transformar el tejido social por medio del ecofeminismo, realizando movimientos sociales como el Chipko o el de Adivasi, con los cuales pueda confrontar y mitigar la discriminación social por carácter de ingresos/pobreza 
o por los bienes que compra o por los que no puede comprar. Haciendo sentir como parte de la sociedad civil a los y las excombatientes.

Para hacernos una idea de que esto es posible, podemos mirar la experiencia de vida de Leonor Esguerra, que pasó de ser una monja a una guerrillera y de ahí nuevamente a la integración a la vida civil en 1994; y quien actualmente vive promoviendo el liderazgo femenino, promoviendo la paz y el cambio favorable para Colombia (Carriquiry, 2011)

Recordando siempre, que las emociones y las pasiones ayudan a tener un control social, y que así como son usadas en las publicidades para vender un producto, también pueden ser usadas para vender una idea, un pensamiento, una forma de vida en un contexto de paz.

\section{Conclusiones y recomendaciones}

En síntesis, se plantea que las empresas deben primero identificar las necesidades del consumidor, aspecto que es muy diferente del famoso dicho que dice "al cliente se le crean las necesidades", realmente no es crearla porque esto es lo que nos lleva a un tiempo de consumismo desmedido sin sentido y tan agresivo con nuestro medio ambiente. Lo que en verdad se debe realizar es auscultar las necesidades para posteriormente satisfacerlas con un producto eficiente. Ya que de esta forma se evitará comprar y botar a la basura porque no cumplió las expectativas, y comprar uno nuevo, y volverlo a botar a la basura, siguiendo con este ciclo que hace parte del sistema de la economía lineal.

Las empresas al introducir un producto al mercado deben recordar que el cliente debe quedar satisfecho, pues esto es la base del éxito empresarial "El client, i per tant el consumidor, és la base del creixement empresarial en un context de consum i gestió integrat" (Roco).
Se destacan los aportes que desde el género femenino se pueden dar para una sociedad en torno al posconflicto. A través de cambios en sus hábitos de consumo, y desde el ecofeminismo, tal y como se ha explicado en detalle en el apartado de posconflicto.

También se realiza un llamado a la sociedad en general para que se pregunten al momento de comprar cualquier producto, ¿es esto lo que verdaderamente quiero?, ¿cumple mis expectativas?, ¿lo compro porque me gusta o porque todos tienen uno, todos lo usan y yo necesito encajar?

Es verdad que siempre seremos parte de este sistema, mientras el modelo capitalista funcione, sin embargo, podemos elegir qué comprar, cuándo comprar, dónde comprar, cada cuánto comprar y lo más importante, el por qué comprarlo. Recordando que el precio que se paga por el producto no es lo único que se da a cambio, ya que cada vez que adquirimos algo sin necesidad enviamos una parte de nuestro planeta a la destrucción.

Para finalizar, voy a referirme brevemente a un caso en especial que me ocurrió durante el estudio etnográfico, donde una mujer joven se tornó escéptica sobre el porqué ella debía contribuir con el mejoramiento del mundo, argumentando que nadie más lo hacía. Tuve la opción de disponerme a contarle las múltiples acciones que mucha gente realiza para contribuir con el medio ambiente, sin embargo, hubiera podido pasar horas contando estas acciones y ella hubiera seguido igual, entonces, prefiero contestar con un argumento egoísta sobre el por qué el ecofeminismo es importante, relacionado con los hábitos de consumo para las mujeres:

Las mujeres deben tener control sobre lo que compran, teniendo un carácter ecológico, y no solo por el medio ambien- 
te, por la contaminación actual vivida a través del esmog o niebla contaminante, o para dejarles un mundo mejor a sus hijos y nietos, sino también desde un punto totalmente egoísta, pensando en sí misma, que es totalmente válido, pues es algo del ahora, del presente. Si la mujer no controla los impulsos de compra (sobre todo en bienes innecesarios que hacen parte de la economía lineal) se está exponiendo a sí misma a tener problemas de salud, específicamente en la patología, cáncer de mama, incrementada por los xenoestrogenos (sustancias químicas tóxicas) que están presentes en gran cantidad de productos, incluidos en aquellos fabricados con plásticos (Puelo, 2008).

Sin embargo, este no es un llamado solo para las consumidoras, también lo es para las empresas, compañías y fábricas, quienes usan este tipo de sustancias tóxicas en sus productos, y que aun y cuando las mujeres decidan no comprarlo estaría afectando a aquellas que trabajan en dichos lugares. Por esto, se hace pertinente que las empresas tengan en cuenta su huella de carbono y su responsabilidad social y medio ambiental para con lo mismo, que no se limita a recursos económicos.

\section{Referencias}

Assosiation for Women's Rights im Development. (2004). Interseccionalidad: una herramienta para la justicia de género y la justicia económica. AWID, 1-8.

Belz, F. M., Peattie, K., \& Galí, J. (2013). Marketing de sostenibilidad. Profit Editorial.

Borrás, I. (2000). Psicología económica y del comportamiento del consumidor. Editorial UOC.

Carriquiry, L. E. (2011). La busqueda.

Figueredo, M., \& Rozo, E. (2002). Legislación turística en Colombia. Bogotá: Universidad Externado de Colombia.

García, S., \& Thomas, H. (2011). Psicologia aplicada a la publicidad. Dykinson.

Guerra Manzo, E; (2010). Las teorías sociológicas de Pierre Bourdieu y Norbert Elias: los conceptos de campo social y habitus. Estudios Sociológicos, XXVIII 383-409. Recuperado de http://www.redalyc.org/ articulo.oa?id $=59820673003$

Medina-Vincent, M. (2013). Evolución del ecofeminismo. Fòrum de recerca, 17, 53-69.

Osorio, D. A. (2013). Análisis del segmento de los baby boomers a partir delportafolio de las marcas colombianas y aporte para mejorar su implementación en la publicidad. Santiago de Cali: Universidad Autónoma de Occidente.

Prieto, J. E. (2011). Merchandising: la seducción desde el punto de venta. Elibro.

Puelo, A. H. (2008). Escuchar la vida: Mensajes para una alternativa necesaria y posible.

Puelo, A. H. (2008). Libertad, igualdad, sostenibilidad. Por un ecofeminismo ilustrado. Revista de Filosofia Moral y Política, 39-59.

Roco, M. (s.f.). Cap a una economia circular. Raco.cat.

Rpp Noticias. (2013). EE. UU. crea mapa que muestra pérdida mundial de bosques y junglas. Rpp Noticias.

Santamarina, \& Marinas. (1994). Comportamiento del consumidor. 